\title{
A COINCIDENCE THEOREM RELATED TO THE BORSUK-ULAM THEOREM
}

\author{
FRED COHEN AND J. E. CONNETT
}

\begin{abstract}
A coincidence theorem generalizing the classical result of Borsuk on maps of $S^{n}$ into $R^{n}$ is proved, in which the antipodal map is replaced by a $Z_{p}$-action on a space which is $(n-1)(p-1)$-connected.
\end{abstract}

The main result is:

THEOREM 1. Let $X$ be a Hausdorff space which supports a free $Z_{p}$-action, and $f: X \rightarrow R^{n}$ a continuous map, $n \geqq 2$. If $X$ is $(n-1)(p-1)$-connected, then there exists $x \in X$ and $g \in Z_{p}, g \neq$ identity, such that $f(x)=f(g x)$.

We observe that if $p=2$, then Theorem 1 is a restatement of the classical Borsuk-Ulam theorem.

The case $n=2$ has been studied by the second author [3], using the fact that Artin's braid groups have no elements of finite order. For this case it suffices to assume only that $\pi_{1}(X)$ is a torsion group.

The cases $n>2$ require a bit more geometry. We recall the definition of the configuration space $F(M, j)$, of $j$ distinct points in a space $M$ : $F(M, j)$ is the subspace of $M^{j}$ given by $\left\{\left\langle x_{1}, \cdots, x_{j}\right\rangle \mid x_{i} \in M, x_{i} \neq x_{j}\right.$ if $i \neq j\}$. The spaces $F(M, j)$ have been studied by Fadell and Neuwirth [4]. Evidently. $\Sigma_{j}$, the symmetric group on $j$ letters, acts freely on $F(M, j)$ by permutation of coordinates.

We define $F\left(R^{\infty}, j\right)$ to be inj $\lim _{n} F\left(R^{n}, j\right)$, where $F\left(R^{n}, j\right) \subset F\left(R^{n+1}, j\right)$ is given by the standard inclusion of $R^{n}$ in $R^{n+1}$. By [2], $F\left(R^{\infty}, j\right)$ is contractible. Since $Z_{p}$, the cyclic group of order $p$, acts on $F\left(R^{n}, p\right)$ and $F\left(R^{\infty}, p\right)$ via the action given by a homomorphism $Z_{p} \rightarrow \Sigma_{p}$ which sends $1 \in Z_{p}$ to the cycle $(1,2, \cdots, p)$, it follows that $F\left(R^{\infty}, p\right) / Z_{p}$ is a $K\left(Z_{p}, 1\right)$ space. We shall assume without loss of generality that $p$ in the hypothesis of Theorem 1 is prime.

With these preliminaries, we state the main lemma; the lemma's proof is deferred till after the proof of Theorem 1.

Lemma 2. $H^{i}\left(F\left(R^{n}, p\right) / Z_{p} ; Z_{p}\right)=0$ if $i>(n-1)(p-1)$.

Received by the editors August 8, 1973.

AMS (MOS) subject classifications (1970). Primary 55C20, 55C35.

(C) American Mathematical Society 1974 
Proof OF Theorem 1. Let $\sigma$ denote the generator of the cyclic group $Z_{p}$. We suppose that Theorem 1 is false, i.e., $f(x) \neq f\left(\sigma^{i} x\right)$ for all $x \in X$ and all $i$ such that $1 \leqq i \leqq p-1$. Define $\psi: X \rightarrow F\left(R^{n}, p\right)$ by the formula $\psi(x)=$ $\left\langle f(x), f(\sigma x), \cdots, f\left(\sigma^{p-1} x\right)\right\rangle$. Clearly $\psi$ is a continuous, $Z_{p}$-equivariant map. Since $X$ is Hausdorff, $\psi$ induces a map of covering spaces:

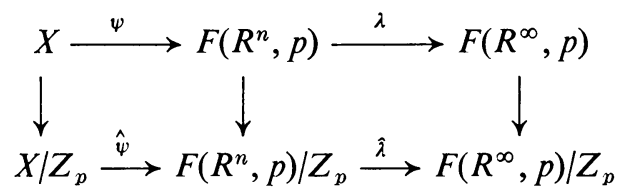

(vertical arrows represent quotient maps; $\lambda$ and $\hat{\lambda}$ are the obvious inclusions). By naturality of the spectral sequence for a covering [1, pp. $355-358]$ and the fact that $X$ is $(n-1)(p-1)$-connected, it follows immediately that $\hat{\psi}^{*} \circ \hat{\lambda}^{*}: H^{*}\left(K\left(Z_{p}, 1\right) ; Z_{p}\right) \rightarrow H^{*}\left(X \mid Z_{p} ; Z_{p}\right)$ is an isomorphism in degrees $\leqq(n-1)(p-1)$ and a monomorphism in degree $(n-1)(p-1)+1$.

It is well known that

$$
\begin{aligned}
H^{*}\left(K\left(Z_{p}, 1\right) ; Z_{p}\right) & =P[u] \quad \text { if } p=2, \\
& =E[u] \otimes P[\beta u] \text { if } p>2,
\end{aligned}
$$

as an algebra, where $P[u]$ denotes the polynomial algebra on a onedimensional class $u, E[u]$ denotes the exterior algebra on a one-dimensional class $u$, and $P[\beta u]$ denotes the polynomial algebra on the Bockstein of $u$ $[1$, p. 252]. (We will consider the cases $p>2$ since the case $p=2$ is analogous and easier.) Hence $\hat{\psi}^{*} \circ \hat{\lambda}^{*}\left(u^{\varepsilon}(\beta u)^{k}\right) \in H^{*}\left(X / Z_{p} ; Z_{p}\right)$ is nonzero provided $\varepsilon=0,1$ and $\varepsilon+2 k \leqq(n-1)(p-1)+1$. But by Lemma $2, \hat{\lambda}^{*}\left(u^{\varepsilon}(\beta u)^{k}\right)=0$ if $\varepsilon+2 k=(n-1)(p-1)+1$, which is a contradiction to our hypothesis that $f(x) \neq f\left(\sigma^{i} x\right)$ for $x \in X, 1 \leqq i \leqq p-1$. This proves Theorem 1 .

We remark that this proof of Theorem 1 is actually a generalization of the proof of the Borsuk-Ulam theorem which relies on the truncated polynomial algebra $H^{*}\left(P^{n} ; Z_{2}\right)$.

Proof of Lemma 2. Let $\left\{E_{r}\right\}$ denote the spectral sequence for the covering whose $E_{2}^{* *}$ term is $H^{*}\left(Z_{p} ; H^{*}\left(F\left(R^{n}, p\right) ; Z_{p}\right)\right)$ and which converges to $H^{*}\left(F\left(R^{n}, p\right) / Z_{p} ; Z_{p}\right)$. By Theorem IV of [2] (the 'vanishing theorem'), $E_{2}^{s, t}=0$ if $s>0$ and $t \neq 0,(n-1)(p-1)$ or $t>(n-1)(p-1)$. By the periodicity argument in [2], it is easy to see that no classes of total degree greater than $(n-1)(p-1)$ can survive to $E_{\infty}^{* *}$. This proves Lemma 2.

Remarks. Another generalization of the Borsuk-Ulam Theorem has been proved by Munkholm [5], whose result implies that if $f: S^{k} \rightarrow R^{n}$ is continuous and $\sigma: S^{k} \rightarrow S^{k}$ generates a $Z_{p}$-action on $S^{k}$ and $k \geqq n(p-1)$, 
then there exists $x \in S^{k}$ such that $f(x)=f\left(\sigma^{i} x\right)$ for all $i, 1 \leqq i \leqq p-1$. Thus Munkholm's result requires a stronger hypothesis than our theorem, but also yields a stronger conclusion.

For $p \geqq 3$ and $n \geqq 1$, one easily finds continuous maps $f: S^{2 n-1} \rightarrow R^{n+1}$ for which $f(x) \neq f\left(\sigma^{i} x\right)$ for any $i, 1 \leqq i \leqq p-1$, and any $x \in S^{2 n-1}$. This shows that Theorem 1 is best possible for $p=3$, in the sense that the hypothesis that $X$ is $(n-1)(p-1)$-connected cannot be weakened.

\section{REFERENCES}

1. H. Cartan and S. Eilenberg, Homological algebra, Princeton Univ. Press, Princeton, N.J., 1956. MR 17, 1040.

2. F. Cohen, Cohomology of braid spaces, Bull. Amer. Math. Soc. 79 (1973), 763-766.

3. J. E. Connett, A generalization of the Borsuk-Ulam theorem, J. London Math. Soc. (2) 7 (1973), 64-66.

4. E. Fadell and L. Neuwirth, Configuration spaces, Math. Scand. 10 (1962), 111-118. MR 25 \#4537.

5. H. J. Munkholm, Borsuk-Ulam type theorems for proper $Z_{p}$-actions on $(\bmod p$ homology) n-spheres, Math. Scand. 24 (1969), 167-185. MR 41 \#2672.

Department of Mathematics, Northern Illinois University, DeKalb, Illinois 60115 